\title{
Constraints on Bars in the Local Universe from 5000 SDSS Galaxies
}

\author{
Fabio D. Barazza, Shardha Jogee, and Irina Marinova \\ Department of Astronomy, University of Texas at Austin, 1 University Station C1400, Asutin, \\ TX 78712-0259, USA \\ email: barazza@astro.as.utexas.edu
}

\begin{abstract}
We present the first study of bars in the local Universe, based on the Sloan Digitized Sky Survey (SDSS). The large sample of $\sim 5000$ local galaxies provides the largest study to date of local bars and minimizes the effect of cosmic variance. The sample galaxies have $M_{g} \leqslant-18.5$ mag and cover the redshift range $0.01 \leqslant z<0.04$. We use a color cut in the color-magnitude diagram and the Sérsic index $n$ to identify disk galaxies. We characterize bars and disks using $r$ band images and the method of iterative ellipse fits and quantitative criteria developed in Jogee at al. (2004, ApJL, 615, L105). After excluding highly inclined $\left(i>60^{\circ}\right)$ systems our results are: (1) the optical ( $r$-band) fraction of barred galaxies among local disk galaxies is $43 \%$ (Figure 1, left panel), which confirms the ubiquity of local bars, in agreement with other optical studies based on smaller samples (e.g. Eskridge et al. 2000, AJ, 119, 536, Marinova \& Jogee 2006, astro-ph/0608039); (2) the optical bar fraction rises for bluer galaxies, suggesting a relation between bars and star formation (Figure 1, middle panel); (3) preliminary analyzes suggest that the optical bar fraction increases steeply with the galaxy effective radius $\left(r_{\text {eff }}\right.$, Figure 1, right panel); (4) the optical bar fraction at $z \sim 0$ is $\sim 35 \%$ for bright disks $\left(M_{g} \leqslant-19.3 \mathrm{mag}\right.$ ) and strong (bar ellipticity $>0.4$ ), large-scale (bar semi-major axis $>1.5 \mathrm{kpc}$ ) bars, which is comparable to the value of $\sim 30 \pm 6 \%$ reported earlier (Jogee et al. 2004) for similar disks and bars at $z \sim 0.2-1.0$.
\end{abstract}

Keywords. galaxies: evolution, galaxies: formation, galaxies: structure
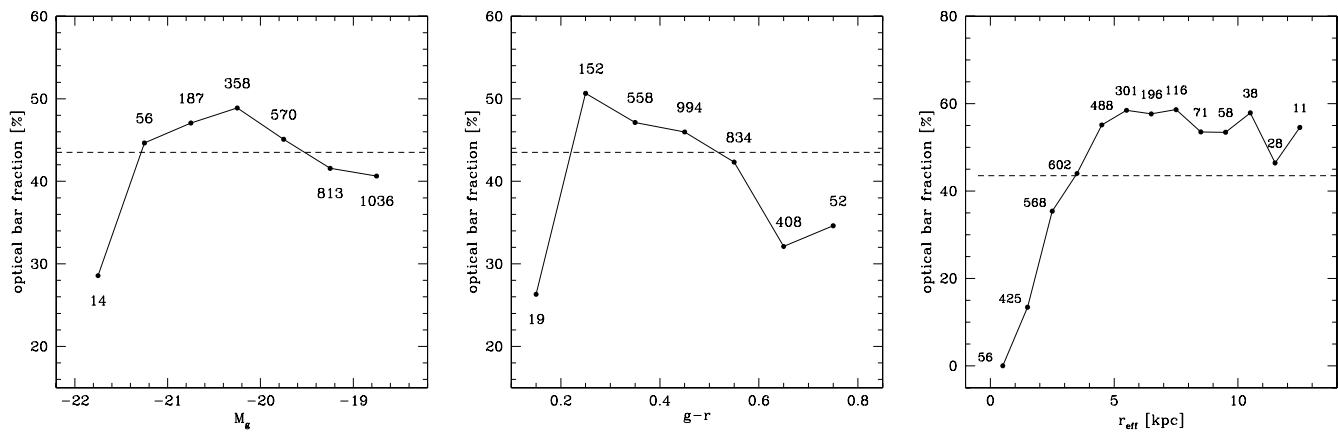

Figure 1. The optical bar fraction as a function of $M_{g}$ (left panel), $g-r$ color (middle panel), and $r_{\text {eff }}$ (right panel). The number next to each point denotes the total number of galaxies in the corresponding bin. The dashed line indicates the total optical bar fraction. Only bins with more than 10 objects are shown.

\section{Acknowledgements}

We acknowledge support from NSF grant AST-0607748, NASA LTSA grant NAG513063, and HST grant GO-10395. 